\title{
Comparison of Carbon Monoxide Levels During Heating of Ice and Water to Boiling Point With a Camping Stove
}

\author{
Simon Leigh-Smith, MBChB, MRCGP, FRCSEd (A\&E); Ian Watt; Angus McFadyen, BSc, MSc, DipSAD, \\ Cmath, MIMA, FSS; Stan Grant, PhD, MSc, BEd DPE \\ From the Defence Medical Services, UK (Dr Leigh-Smith); the Institute of Biomedical and Life Sciences, University of Glasgow, Glasgow, \\ Scotland (Mr Watt and Dr Grant); and Glasgow Caledonian University, Glasgow, Scotland (Mr McFadyen).
}

\begin{abstract}
Objectives.-To determine whether using a camping stove to bring a pan of ice to boiling point produces higher carbon monoxide $(\mathrm{CO})$ concentration than would bringing a pan of water to boiling point. The hypothesis was that ice would cause greater $\mathrm{CO}$ concentration because of its greater flamecooling effect and, consequently, more incomplete combustion.

Method.- This was a randomized, prospective observational study. After an initial pilot study, CO concentration was monitored during 10 trials for each of ice and water. A partially ventilated $200-\mathrm{L}$ cardboard box model was developed and then used inside a chamber at $-6^{\circ} \mathrm{C}$. Ice temperature and volume, water temperature and volume, pan size, and flame characteristics were all standardized. Temperature of the heated medium was monitored to determine time to boiling point. Carbon monoxide concentration was monitored every 30 seconds for the first 3 minutes, then every minute until the end of each 10-minute trial.

Results.-There was no significant difference $(P>.05)$ in CO production levels between ice and water. Each achieved a similar mean plateau level of $\sim 400 \mathrm{ppm}$ CO concentration with a similar rate of rise. However, significantly higher $(P=.014) \mathrm{CO}$ concentration occurred at 4 and 5 minutes when the flame underwent a yellow flare; this occurred only on 3 occasions when ice was the medium.

Conclusion.- There were no significant differences for CO production between bringing a pan of ice or water to boiling point. In a small number of ice trials, the presence of a yellow flame resulted in high $\mathrm{CO}$ concentration. Yellow flares might occur more often with ice or snow melting, but this has not been proven.
\end{abstract}

Key words: carbon monoxide, stove, camp, poisoning

\section{Introduction}

Melting snow and ice is a common activity for military personnel, explorers, and climbers and can result in a prolonged period when the stove flame is in contact with a cold pan. If carbon monoxide (CO) production levels were high during this period, it would represent a particularly dangerous time, especially if the tent or snow cave had limited ventilation.

Although many accounts describe both fatal and nonfatal CO poisoning inside tents ${ }^{1-10}$ (Ministry of Defence, unpublished data, 1993), little detailed information exists on the conditions relating to these incidents. Cooking may produce higher $\mathrm{CO}$ concentration in snow holes and

Corresponding author: Simon Leigh-Smith, Institute of Naval Medicine, Monckton House, Alverstoke, Gosport, Hants, PO12 2DL, UK (e-mail: simonlsuk@aol.com). tents (D. Smith, unpublished data) ${ }^{11-13}$ and raised carboxy haemoglobin concentrations of tent occupants 5 compared with those merely using the stove as a heater. Some observational studies on $\mathrm{CO}$ production from camping stoves have concluded that $\mathrm{CO}$ production is lowest with a freely burning flame and is increased by pan contact with the flame while cooking or melting snow and ice (D. Smith, unpublished data). ${ }^{5,13-16}$ Higher $\mathrm{CO}$ concentration with cooking may be a result of flame cooling, with elevated levels of incomplete combustion (D. Smith, unpublished data). ${ }^{12,14}$

We therefore hypothesized that CO production would be higher when taking a pan of ice to boiling than when taking a pan of water to boiling, and we developed a model to test this hypothesis. Our aim was to compare $\mathrm{CO}$ concentration during heating of ice and water to boiling point with a camping stove. Although the gas- 
eous volumes involved in this model are much smaller than a tent or snow cave, we believed it was appropriate because the experiment was comparing $\mathrm{CO}$ production under standardized conditions to allow comparison of 2 variables.

\section{Methods}

\section{PILOT STUDY}

This study consisted of trials that were performed to develop the experimental model and ensure standardization of all extraneous factors before testing the main hypothesis. The factors examined included type of stove, fuel, level of ventilation, and pan size. Details are included here and in the "Results" section; although they lack full scientific rigor, they may stimulate further research.

Kerosene fuel was initially used because it has been shown to produce the highest CO concentration in camping stoves. ${ }^{10}$ However, it proved impossible to standardize the flame with kerosene in an MSR XGK II stove because it burned erratically, often with a dirty, sooty, yellow flame, and kerosene would not burn at all in a Coleman Peak stove. A Coleman Peak stove with Coleman fuel gave consistent, predictable maximum blue flames and, despite being a very clean burning fuel, ${ }^{10}$ still produced detectable and variable $\mathrm{CO}$ concentration in different conditions.

Although $\mathrm{CO}$ production can be increased with a closed system as a result of inadequate air supply and exhaust ventilation, thereby resulting in low oxygen $\left(\mathrm{O}_{2}\right)$ and high carbon dioxide $\left(\mathrm{CO}_{2}\right),{ }^{14}$ the disadvantage is that the flame becomes extinguished. ${ }^{15}$ This problem can be overcome by artificially inputting $\mathrm{O}_{2}$ into the system, but difficulties arise when deciding how much to add to the combustion mixture. Therefore, initial pilot trials used a nonventilated 200-L cardboard box at ambient temperature $\left(\sim 15^{\circ} \mathrm{C}\right)$ - similar to that used successfully by Schwartz et al. ${ }^{10}$ However, this nonventilated box model led to frequent and unpredictable occurrences of either the maximum blue flame undergoing a yellow flare or the stove self-extinguishing. These were detected by the flame becoming quiet either with rapidly rising and extremely high $\mathrm{CO}$ concentration (yellow flares) or with rapidly falling $\mathrm{CO}$ concentration (self-extinguishing) from a presumed lack of $\mathrm{O}_{2}$.

Further pilot trials developed the partially ventilated box model that was used to test the main hypothesis (Figure 1). This model allowed the maximum blue flame (Figure 2) to burn indefinitely with no self-extinction and give consistent $\mathrm{CO}$ concentration for standardized conditions yet still yield different $\mathrm{CO}$ concentration in varying experimental conditions. Although less frequent with this model, the blue flame was occasionally witnessed to suddenly flare yellow (Figures 3 and 4), followed by fuel leaking from the stove jets and the stove becoming depressurized. During some of the yellow flares, water was seen dripping onto the stove element and was a possible cause. All steps were taken to try to prevent this by ensuring that the pan base was level and allowing any water to drop off the edge of the pan outside the stove element rather than off the pan center into the flame jets. However, this did not completely eradicate the problem, and the reason why it happened remains unclear.

Once this model had been successfully developed, 3 trials for each of stove alone, water, ice, and boiling water were run to gain an impression of how these differences affected $\mathrm{CO}$ production and give guidance for the power calculation.

\section{MAIN STUDY}

The main study was a randomized, prospective observational study performed at sea level. It tested the null hypothesis that there is no difference in $\mathrm{CO}$ production between ice and water when bringing a pan of each medium to boiling point on a camping stove under standardized conditions.

A $200-\mathrm{L}(100 \times 45 \times 45 \mathrm{~cm}), 1-\mathrm{cm}$ thick cardboard box that had a $900-\mathrm{cm}^{2}(20-\mathrm{cm}$ high $\times 45-\mathrm{cm}$ wide $)$ area of ventilation on 1 side at floor level was used, and convection was minimized by having the other 3 sides of the box in contact with the ground. This ventilation port allowed $\mathrm{O}_{2}$ influx for combustion at the bottom of the box along with $\mathrm{CO}$ monitoring at the top of the box, as $\mathrm{CO}$ tends to rise as a result of its properties as a hot combustion gas and its lower density (molecular weights: $\mathrm{CO}=28$, nitrogen $=28, \mathrm{O}_{2}=32, \mathrm{CO}_{2}=$ 44). As the box became full of hot combustion gases, the height of this ventilation port $(20 \mathrm{~cm})$ relative to the height of the stove jets $(13 \mathrm{~cm})$ allowed exiting of these gases without extinguishing the stove.

The box was placed on end inside a $24000-\mathrm{L}$ cold chamber and maintained at $-6^{\circ} \mathrm{C} \pm 2{ }^{\circ} \mathrm{C}$. This was done because lower ambient temperatures may lead to higher CO concentration. ${ }^{14}$ The stove was positioned in the center underneath this box. Aluminium pans were used, with a base diameter of $165 \mathrm{~mm}$ and volume of $1 \mathrm{~L}$ of medium. The medium starting temperature was $5^{\circ} \mathrm{C} \pm$ $2^{\circ} \mathrm{C}$ for the water and $-7^{\circ} \mathrm{C} \pm 2^{\circ} \mathrm{C}$ for the ice, which was frozen into the pan as a solid block in a commercial freezer.

A K-type thermocouple, connected to a Comark KM1242 logger, sensed and recorded the medium temperature $3 \mathrm{~cm}$ above the center of the pan base and was 


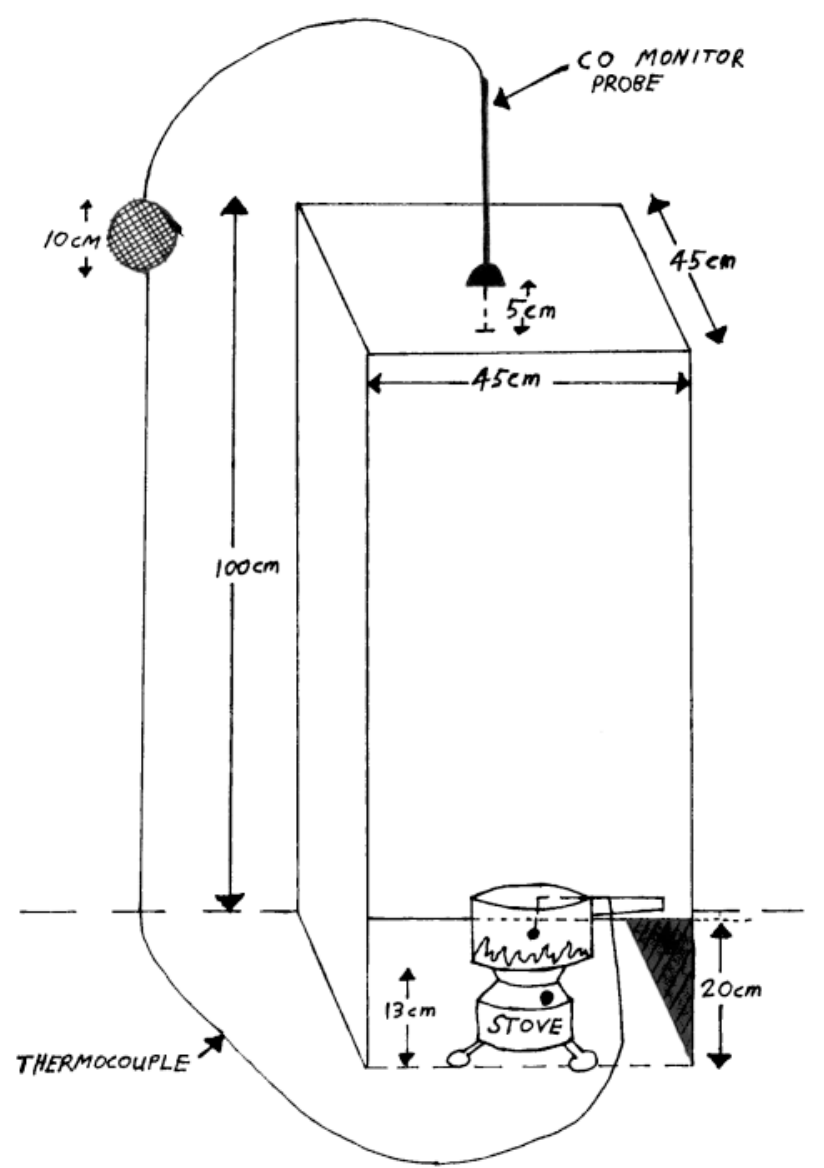

Figure 1. Partially ventilated box model.

prefrozen into the ice pans. The remote sensor of a Neotronics CO101 penetrated the top of the box to a depth of $5 \mathrm{~cm}$ ( $82 \mathrm{~cm}$ above the flame). This gave continuous $\mathrm{CO}$ concentration readouts at the top of the cardboard box, up to a concentration of $4000 \mathrm{ppm}$. Carbon monoxide concentration and temperature monitoring was performed outside the chamber with remote monitors and extended sensors placed through a $10-\mathrm{cm}$ diameter hole in the chamber wall. Before each trial, the 200-L box and environmental chamber were ventilated to 0 ppm CO concentration. Visual monitoring of the stove and flame was performed through the ventilation port of the box at the start and end of each trial but also by 1 investigator re-entering the chamber if $\mathrm{CO}$ concentration rose uncharacteristically high during the trial-as what generally happened with yellow flares.

For each trial, the Coleman Peak stove was refilled with Coleman fuel and had a standardized priming sequence: 30 pumps, light, 30 pumps, set control lever to maximum, burn for 5 minutes, 30 pumps. This was done to preheat the stove and standardize the flame characteristics to maximum blue before commencing the trial.

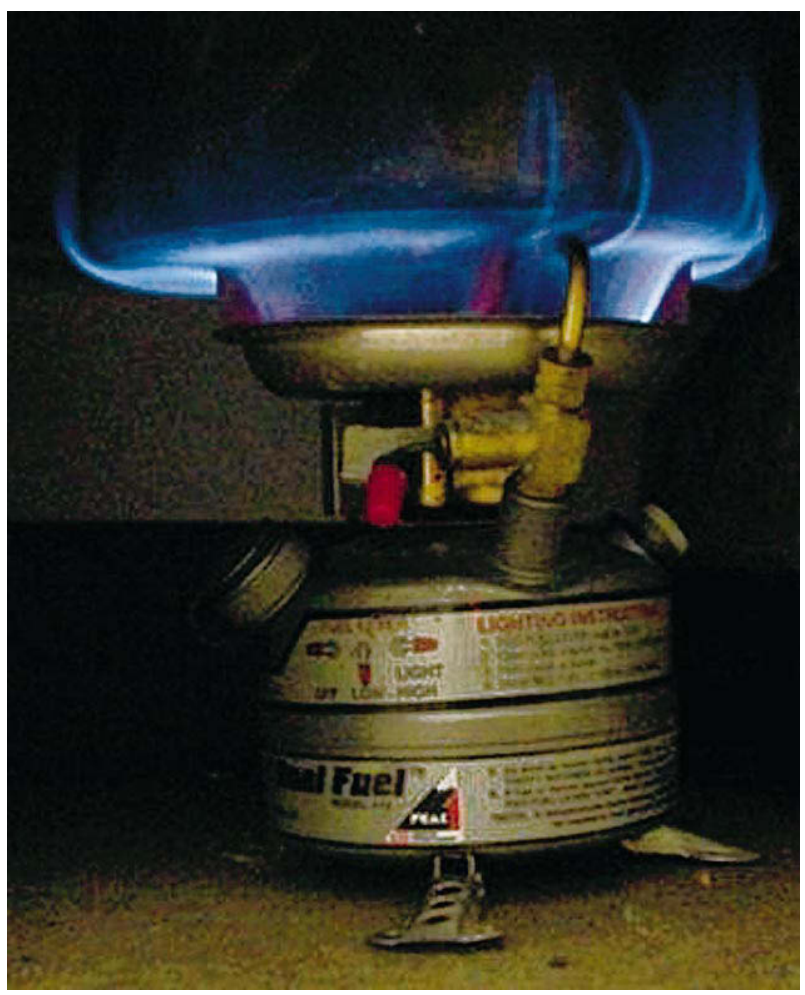

Figure 2. Maximum blue flame, yellow flame $\times 2$.

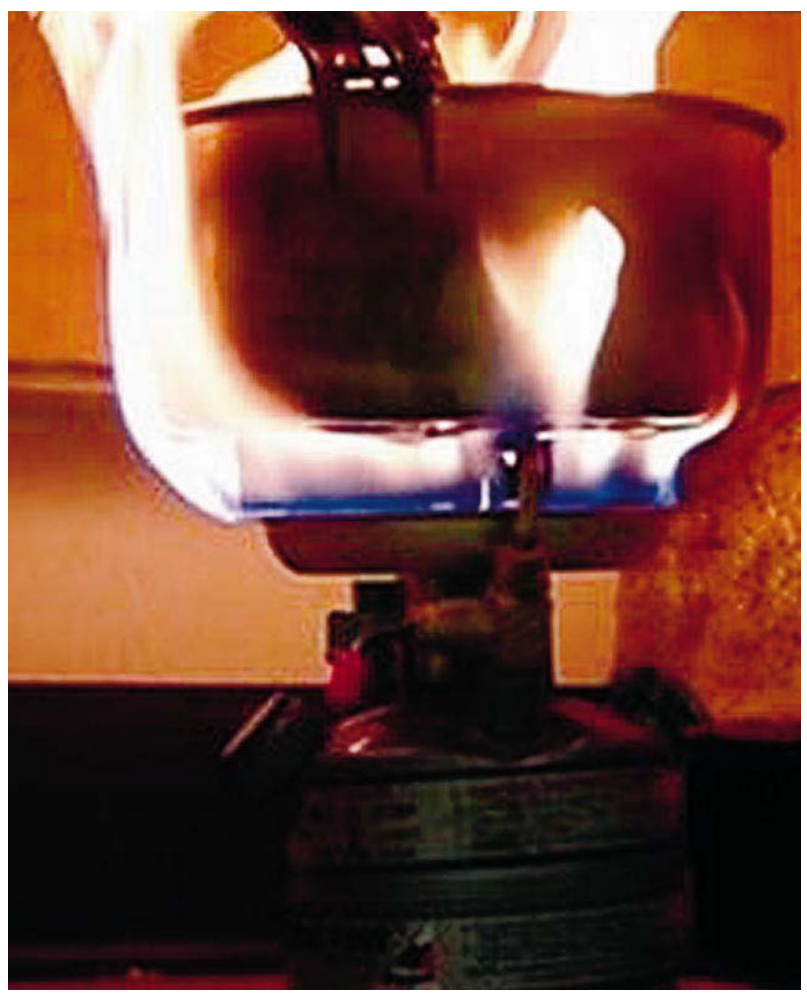

Figure 3. Maximum blue flame, yellow flame $\times 2$. 


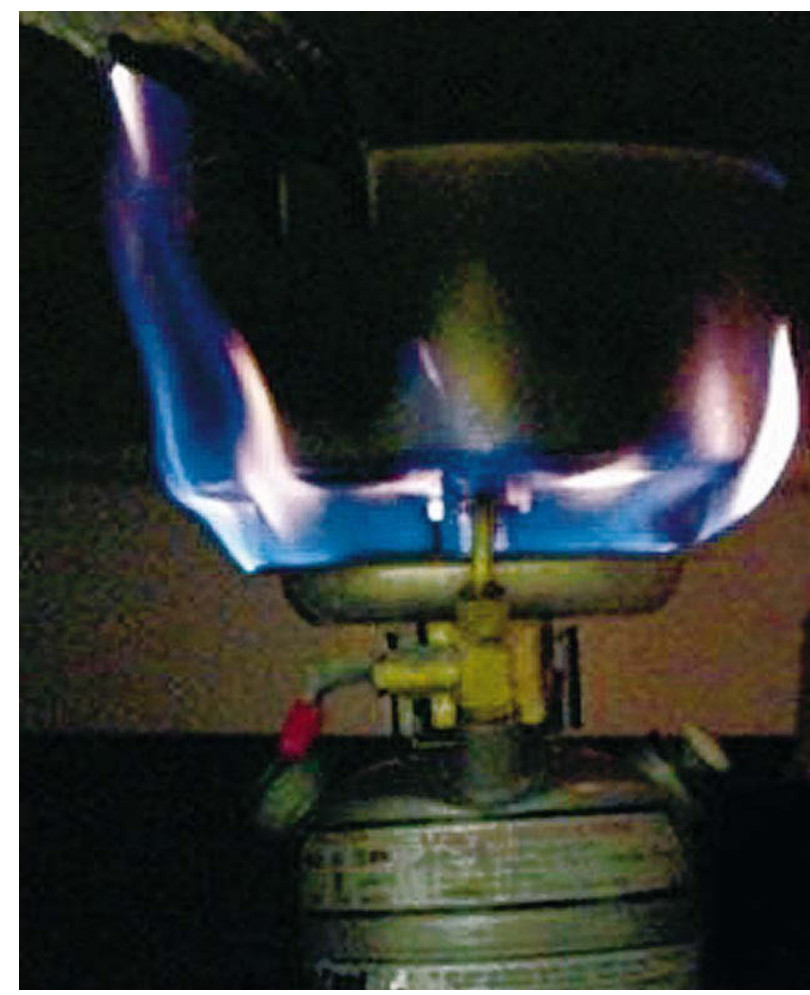

Figure 4. Maximum blue flame, yellow flame $\times 2$.

Although a low flame produces the highest $\mathrm{CO}$ concentration, ${ }^{12}$ a maximum blue flame was adopted for this experiment, for that would usually set the conditions for bringing water to boiling point more standardized than with a low-flame setting. A blue flame also indicates adequate $\mathrm{O}_{2}$ for combustion because a flame will not burn blue in "foul" air conditions. 5

Monitoring time started for each trial when the pan was placed onto the stove. Ten trials for each medium were run in random order. Carbon monoxide concentration was monitored every 30 seconds for the first 3 minutes and then every minute until ceasing each trial at 10 minutes.

\section{STATISTICAL ANALYSIS}

Results were analyzed by the Minitab statistical package $^{17}$ to detect any differences between water and ice, and the findings of high $\mathrm{CO}$ concentration with yellow flares was also included in the analysis. Because of the large differences in variation, the Kruskal-Wallis nonparametric analysis of variance was used initially. The Mann-Whitney $U$ test with Bonferroni correction $(\alpha=$ $.05 / 3=.017$ ) due to multiple testing was used for follow-up analysis.

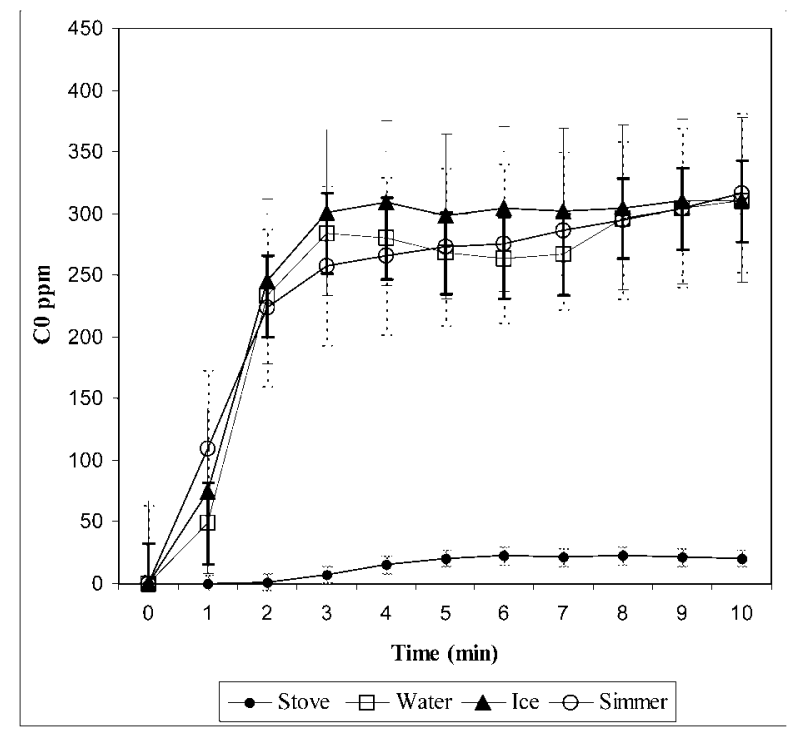

Figure 5. Pilot study-ventilated box model. Means of 3 replicates for each of the 4 conditions $\pm 1 \mathrm{SD}$.

\section{Results}

\section{PILOT STUDY}

Figure 5 shows the results of the 3 trials for each of stove alone, water, ice, and boiling water, which, with the exception of ambient temperature and pan diameter ( 2 pans used; $155 \mathrm{~mm}$ and $165 \mathrm{~mm}$ ), were standardized with respect to all the methodology in the main study. Because it was uncertain whether pan diameter was, in fact, the cause of the large variance, a power calculation for the main standardized trials was performed with the clinical difference to be detected taken as $200 \mathrm{ppm}$. This is a relatively arbitrary figure, for the methodology is designed to simply detect relative differences, and application of these to clinical meaning is difficult. However, because the plateau $\mathrm{CO}$ concentration was around $300 \mathrm{ppm}$, an increase of $67 \%$ was thought to be a reasonable cut off for delineating significance. On the basis of this, an SD of $80 \mathrm{ppm}, \alpha 5 \%$, and power $80 \%$, a minimum of 4 replicates was required to demonstrate this difference. The actual study involved 10 trials for each variable to ensure that smaller differences were not missed and a type $2 /$ beta error was not introduced.

\section{MAIN STUDY}

The variance (Table 1) for these trials is similar to that seen in the less standardized pilot trials, again suggesting greater variation with melting ice.

Figure 6 shows the means $\pm 1 \mathrm{SD}$ of 10 ice and 10 water trials in the cold chamber along with the means $\pm 1 \mathrm{SD}$ of 3 yellow flares. Each of these yellow flares 
Table 1. Main study: SD for different media

\begin{tabular}{lcc}
\hline \multirow{2}{*}{ Medium } & \multicolumn{2}{c}{$\begin{array}{c}\text { SDs for carbon monoxide } \\
\text { concentration (1 min onward), ppm }\end{array}$} \\
\cline { 2 - 3 } & Range of values & Average value \\
\hline Water & $24-49$ & 36 \\
Ice & $42-91$ & 69 \\
Yellow flare & $24-350$ & 167 \\
\hline
\end{tabular}

occurred during an ice trial and are excluded from the statistical analysis of the null hypothesis, as the flame lost its standardization. For this reason, ice had a total of 13 trials. Boiling points are not marked on Figure 6 but were 8.7 minutes $(\mathrm{SD}=0.42$ ) for ice and $4.5 \mathrm{~min}$ utes (no variance) for water.

No differences were detected between water and ice at any time from 30 seconds onwards, with $P>.05$ at each measurement. These results, therefore, do not allow rejection of the initial null hypothesis. A nonsignificant difference was found between 4 and 7 minutes, with $\mathrm{CO}$ concentration higher for ice than for water.

There was, however, a significant difference (all $P$ values of .014) at 4 and 5 minutes for both water and ice (standardized trials with maximum blue flames) when each was compared with yellow flares. For standardized water and ice heating, the rate of rise in CO concentration is similar and both plateau at around $400 \mathrm{ppm}$ after 3 minutes. The rate of rise for yellow flares appears fast-

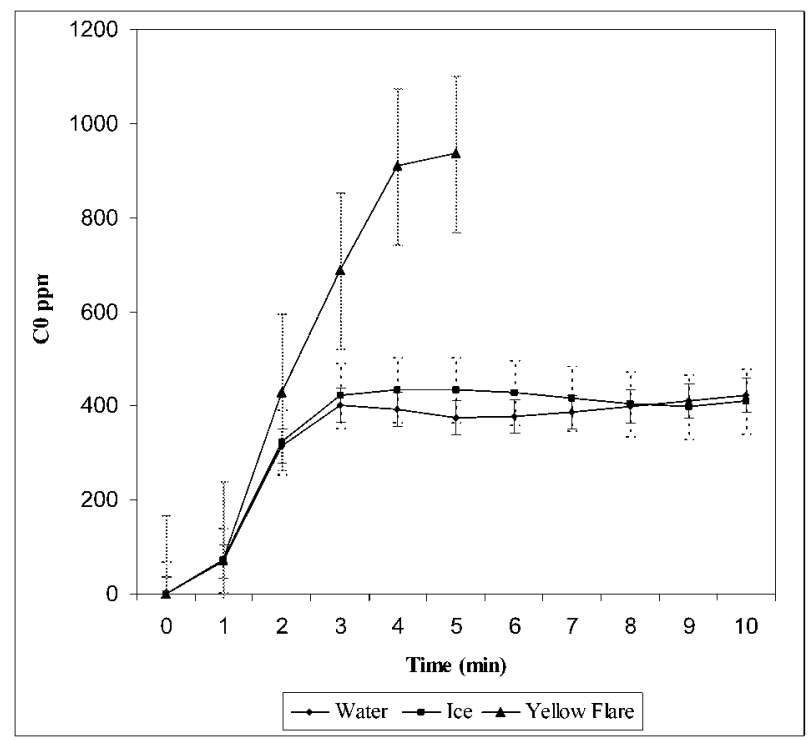

Figure 6. Main study; chamber trials - ice vs water (plus "yellow flares"). Means \pm 1 SD of 10 ice and 10 water trials and means $\pm 1 \mathrm{SD}$ of 3 yellow flares.
Table 2. 95\% CIs for differences in carbon monoxide concentration ppm between yellow flares and heating of ice or water with a maximum blue flame

95\% CI at $4 \min 95 \%$ CI at 5 min

Yellow flare vs water

$$
+360 \text { to }+760+300 \text { to }+1000
$$

Yellow flare vs ice

er, and it was during 1 of these that the maximum $\mathrm{CO}$ concentration of any trial (1340 ppm) was reached, although the mean peak of all yellow flares was $936 \mathrm{ppm}$. Table 2 shows the $95 \%$ CI for the differences in CO concentration between yellow flares and standardized heating (when yellow flares did not occur) of water or ice at these times.

\section{Discussion}

The main study rejects the experimental hypothesis that flame cooling leads to greater $\mathrm{CO}$ production. It has shown no marked increase in $\mathrm{CO}$ production when a pan of very cold ice is placed onto the stove and taken to boiling point. There will be slightly more $\mathrm{CO}$ produced in total when melting ice because it takes longer to boil than does water, but this is believed to be clinically insignificant.

It was during the pilot study that we realized pan diameter may affect $\mathrm{CO}$ production; hence, there is possible slight contamination of these results. This may explain the large variance for all trials except stove alone (Table 3), although similar variances were found during the main study, thereby suggesting this contamination of results is minimal. These results (Figure 5) suggest that there is minimal difference in $\mathrm{CO}$ production between ice, water, and continued heating after boiling (all 300 ppm) while suggesting a large difference between these variables and stove alone $(<30 \mathrm{ppm})$. The findings in

Table 3. Pilot study: SDs for different media

\begin{tabular}{lcc}
\hline \multirow{1}{*}{ Medium } & $\begin{array}{c}\text { SD for carbon monoxide } \\
\text { concentration } \\
(1 \text { min onward }), \text { ppm }\end{array}$ \\
\cline { 2 - 3 } & $\begin{array}{c}\text { Range of } \\
\text { values }\end{array}$ & $\begin{array}{c}\text { Average } \\
\text { value }\end{array}$ \\
\hline Stove alone & $0-12$ & 7 \\
Water & $10-67$ & 33 \\
Ice & $40-91$ & 67 \\
Continued heating after boiling & $34-78$ & 64 \\
\hline
\end{tabular}


the pilot trials of a 10 -fold difference in CO concentration between stove alone and 2 different-sized pans on the flame (with any medium temperature) may suggest that the presence of the pan alone and not the flame cooling raises $\mathrm{CO}$ production. This would suggest that flame dispersal rather than cooling is the cause of higher CO levels with cooking. This observation is quite marked visually when comparing a small blue flame of the stove alone with a large diffuse blue flame that spreads out across the base of the pan and extends up its sides. The finding of higher $\mathrm{CO}$ concentration with low flame settings ${ }^{12}$ supports this explanation as paradoxically higher flame settings cause smaller, hotter flames with camping stoves. Pan diameter may be another independent variable in $\mathrm{CO}$ production from camping stoves, and further discussion of the flame-dispersal hypothesis is given in 2 subsequent articles. ${ }^{18,19}$

The fact that there appears to be greater SDs for ice (supported by evidence from pilot and main trials-Tables 1 and 3) than for water with respect to all its $\mathrm{CO}$ measurements and its boiling point suggest that ice is harder to control experimentally. The reasons for this are unknown but may be worth considering in future work on the subject.

Yellow flaring occurred on 2 occasions with the ventilated box model used in the pilot trials and on 3 occasions with the standardized chamber trials; in each of these occasions, the result was a depressurized stove with a low flame subsequent to the flare. This large yellow flame had a high degree of incomplete combustion and produced significantly higher $\mathrm{CO}$ concentration on each occasion at 4 and 5 minutes compared with water or ice. Ice was the medium on the stove in each of these 5 yellow flares. There may be a correlation between the likelihood of yellow flaring and the medium heated, but the main reason for these flares remains unclear.

\section{LIMITATIONS}

The use of only 1 stove is a potential limitation of this study, and critics may argue that the intermittent yellow flares were attributed to a stove problem. However, a stove burning with a maximum blue flame tends to selfclean by burning any carbon that may have accumulated, and this occurred naturally as part of the stove-priming sequence. We therefore believe that this is unlikely and that the stove was burning efficiently and cleanly at the commencement of each trial.

Another possible limitation of this model design is that the $900-\mathrm{cm}^{2}$ area of ventilation could compensate for any level of $\mathrm{CO}$ production. This might arguably mask any difference among the trials for different mediums and might account for the similar plateau CO con- centration for water and ice. However, this is not thought to be a real problem because of the following:

- Any tendency for this to occur was minimized as discussed in methodology.

- High CO concentration occurred during yellow flares.

- Carbon monoxide concentration was similar to that in a similar but closed cardboard box system. ${ }^{10}$

The nonsignificant difference between 4 and 7 minutes may represent a beta error that indicates we are falsely accepting the null hypothesis of no difference between the water and the ice. This is not the case, though, if our level of clinical significance as used in the initial power calculation is accepted. We also believe this difference is more likely to be related to the difference in flame characteristics at 2 distinct points: onset of boiling and steam surrounding the stove, both of which occur earlier in the water trials. A fall in $\mathrm{CO}$ output is noted soon after boiling for each medium, which then slowly climbs again. Even if this does represent a beta error, the degree of any potential difference when compared with that which occurs with other variables, such as yellow flares, is thought to be of minimal importance. It is also very difficult to decide on the level of clinical significance for a power calculation with this study model, for the significance of such potentially small differences is unknown.

\section{Conclusions}

Flame cooling does not lead to significantly higher production of $\mathrm{CO}$. There will be slightly more $\mathrm{CO}$ produced when bringing a pan of ice to boiling point rather than a pan of water because of the slightly longer time required to boil ice. However, the rate of $\mathrm{CO}$ production is no more than if the pan is kept boiling or simmering for other reasons.

In a small number of ice trials, the presence of a yellow flame resulted in very high levels of CO. Yellow flares might occur more often with ice or snow melting, but this has not been proven. Yellow flames may occur as a result of inadequate $\mathrm{O}_{2}$ for combustion or water dripping onto the stove.

Personnel who use stoves in a poorly ventilated location should be aware that $\mathrm{CO}$ production is higher when a pan is placed on the stove. Yellow flames are associated with very high $\mathrm{CO}$ concentration. Flames should be monitored regularly to check their color, and appropriate action should be taken when they turn yellow. Future work in this area may want to examine the incidence of yellow flares with different media heated or use different stoves in a standardized model such as the one used here. 
Relating the CO levels found in this study to the actual clinical risk within a tent is difficult. A more complete discussion on the risks of $\mathrm{CO}$ poisoning in tents is recommended in the "References" section. ${ }^{19}$

\section{Acknowledgments}

The authors wish to thank the following individuals from the Institute of Naval Medicine, UK: Surgeon Commodore N. Baldock for reference sourcing and funding, Adrian Allsopp and Rob Shayer for concept, Stephen Moore for concept and equipment, and Roger Pethybridge for statistics. The authors also wish to thank the following individuals from the University of Glasgow: Chris Pitt for concept and Martin Watt for concept and references.

\section{References}

1. Foutch RG, Henrichs W. Carbon monoxide poisoning at high altitudes. Am J Emerg Med. 1988;6:596-598.

2. Byrd R. Alone. New York, NY: Adventure Library; 1938.

3. Stefansson W. Unsolved Mysteries of the Arctic. New York, NY: Macmillan; 1939.

4. Irving L, Scholander P, Edwards G. Experiments on carbon monoxide poisoning in tents and snow houses. $J$ Ind Hyg Toxicol. 1942;24:213.

5. Pugh L. Carbon Monoxide hazard in Antarctica. BMJ. 1959;34(5116):192-196.

6. Seibert R. Climbs and expeditions-Alaska. Am Alpine J. 1986;28:139-142.

7. Krakauer J. Into Thin Air-A Personal Account of the Everest Disaster. Chatham, UK: Pan Books; 1997.
8. Girman JR, Chang YL, Hayward SB, et al. Causes of unintentional deaths from carbon monoxide poisonings in California. West J Med. 1998;168:158-165.

9. Centers for Disease Control and Prevention. Carbon monoxide poisoning deaths associated with camping-Georgia, March 1999. JAMA. 1999;282:1326.

10. Schwartz RB, Ledrick DJ, Lindman AL. A comparison of carbon monoxide levels during the use of a multi-fuel camp stove. Wilderness Environ Med. 2001;12:236-238.

11. Keyes LE, Hamilton RS, Rose JS. Carbon monoxide exposure from cooking in snow caves at high altitude. Wilderness Environ Med. 2001;12:208-212.

12. Harrigan M. A Study of Carbon Monoxide Exposure Amongst Troops During Arctic Training [Master's thesis], 1992.

13. Turner WA, Cohen MA, Moore S, et al. Carbon monoxide exposure in mountaineers on Denali. Alaska Med. 1988; 30:85-90.

14. Henderson Y, Turner J. Carbon monoxide as a hazard of polar exploration. Nature 1940;145:92-95.

15. Westerlung $\mathrm{K}$, von Ubisch $\mathrm{H}$. Carbon monoxide from small camping appliances and from stoves without chimney connection. Nordisk Hygienisk Tidskrift. 1972;53:2633.

16. Prescher KE. Occurrence of carbon monoxide, carbon dioxide and nitrogen oxides during the use of gas stoves [in German]. Schriftenr Ver Wasser Boden Lufthyg. 1982;53: 191-198.

17. Statistical Software [computer program]. Version 13.32 . State College, PA: 2003.

18. Leigh-Smith S, Stevenson R, Watt M, et al. Does pan diameter influence carbon monoxide levels during heating of water to boiling point with a camping stove? Wilderness Environ Med. 2004;15:171-174.

19. Leigh-Smith S. Carbon monoxide poisoning in tents-a review. Wilderness Environ Med. 2004;15:157-163. 